\title{
RISK FACTORS, PREVALENCE AND DIAGNOSIS OF HUTCHISON GILFORD SYNDROME WITH SPECIAL REFERENCE TO CASE REPORTS
}

\author{
SAVITA DEVI'1 , KAMALDEEP SINGH² \\ ${ }^{1}$ Lovely Faculty of Applied Medical Sciences, Lovely Professional University, Punjab, India, ${ }^{2}$ Department of Medical Laboratory Sciences, \\ Ct Group of Institute, Shahpur, Jalandhar, Punjab, India \\ Email: savita14545@gmail.com \\ Received: 22 Nov 2016 Revised and Accepted: 20 Mar 2017
}

\begin{abstract}
Progeria also known Hutchinson-Gilford progeria syndrome (HGPS), is an extremely rare genetic disorder. The prevalence of HGPS is 1 in 4-8 million newborns. Progeria causes premature, rapid aging shortly after birth present within the first year of life. Recently, de novo point mutations in the Lmna gene at position 1824 of the coding sequence have been found in persons with HGPS. Lmna encodes lamin A and C, the A-type lamins, which are an important structural component of the nuclear envelope and play a role in protein processing. The most common HGPS mutation is located at codon 608 (G608G). This mutation responsible for creating a cryptic splice site within exon 11, which deletes a proteolytic cleavage site within the expressed mutant lamin A. In Progeria, gene mutation results in the deletion of a Zmpste24/FACE1 splice site in prelamin A, preventing end terminal cleavage. The result of this point mutation can be observed by the main clinical and radiological features include alopecia, thin skin hypoplasia of nails, loss of subcutaneous fat, and osteolysis. The common symptoms of HGPS is a loss of eyebrows and eyelashes which can observed in early childhood and due to receding hairline and blading can also observed. Generally, this patient has facial character include microganthia (small jaw), craniofacial disproportion, prominent eyes, scalp veins and alopecia (loss of hair), restricted joint mobility and severe premature atherosclerosis. Laboratory findings are unremarkable, with the exception of an increased urinary excretion of hyaluronic acid. There is presently no effective therapy is available for Hutchinson-Gilford progeria syndrome (HGPS) but, it is essential to monitor carefully cardiovascular and cerebrovascular disease So, Treatment usually includes low dose aspirin which helps prevent the atherothrombotic events, stroke and heart attacks by hindering platelet aggregation.
\end{abstract}

Keywords: Progeria, Hutchsion Gliford Syndrome, Rapid Aging, Lmna Gene, Osteolysis, Microganthia

(C) 2017 The Authors. Published by Innovare Academic Sciences Pvt Ltd. This is an open access article under the CC BY license (http://creativecommons.org/licenses/by/4.0/) DOI: http://dx.doi.org/10.22159/ijpps.2017v9i5.16282

\section{INTRODUCTION}

Progeria is derived from Greek word "Pro" which means "before" or "forward" and "geron" means "old person"[1] Progeria is also known as hutchsion gliford syndrome. Hutchsion gliford syndrome are come from two scientist named as Jonathan Hutchsion in 1886 and Hasting Gliford in 1897 who independently and delineated to describe this accelerated aging syndrome. Hence, the condition was later re-named after them as Hutchinson gilford progeria syndrome (HGPS) [2]. HGPS are rare genetic disease which designated by segmental increased aging, which are present during the first year of life was only described more than 100 y ago [3, 4]. HGPS come under the inheritance pattern genetic disorder due to the both Autosomal dominant and Autosomal recessive modes which have been introduced in the inheritance pattern of genetic disorder $[5,6]$. Paternal age effect and lack of consanguinity argue are observed mainly due to the sporadic dominant mutations which are inheritance pattern of genetic disorder [7].

Mutation in lamin gene cause a HGPS, so they called laminopathies, It is most probilibily mutation leading to HGPS due to a de novo point mutation in the lamin A gene which activates the cryptic slice donor site, which is mainly responsible for the excision of the $\mathrm{C}$ terminal part of exon 11 [2]. LMNA is an innovative gene of progeria which encodes both the laminins $\mathrm{A}$ and $\mathrm{C}$ [8]. LMNA gene contain exons 1-12 and mainly both lamin A/C contain 12 exon and due to the an alternative splice site in intron 10, which gives to two different mRNA that codes for pre lamin A and lamin C. Lamin A is different from lamin $\mathrm{C}$ at the $\mathrm{C}$ terminal and it lacks final part of exon 10, 11 and 12 [9]. Pre lamin A is a precursor molecule for the synthesis of Lamin A, which contain 664 amino acid proteins with a molecular weight of $70 \mathrm{KDa}$. CAAX box motif is present at the $\mathrm{C}$ terminus of the Lamin A. [10]. Lamin C is slightly smaller than Lmain A lamin $\mathrm{C}$ having a 574 amino acids and weight of 65KDa [11]. LMNA is structure and functional components of the nuclear lamina, whose mainly function is a structural support and it is necessary for the replication of DNA and RNA transcription [8]. HGPS person having maximum chance to survival is $13 \mathrm{y}$, somehow rare cases upto their late teens and early twenties, and rare person may reach to forties [12]. progeria is confederated with several character of premature aging like growth retardation, characteristics facies, restricted joint mobility, prominent eyes and severe premature atherosclerosis. Some other clinical character, may also observed like as craniofacial disproportion, micrognathia, prominent scalpveins, scalp alopecia, wrinkled skin, protruding ears, nail dystrophy, mid facial cyanosis, and a sculpted nose at birth [7].

\section{Epidemiology}

HGPS is a very rare genetic disorder the prevalence of HGPS is 1 in 4-8 million newborns [13]. HGPS equally affected both sexes and all races [14]. The estimated incidence of progeria constant all over the world showing no gender, geographical or ethnic predisposition. Currently, 200-250 children living with progeria are observed all over the worldwide at any one time. At the present time about 114 children with HGPS are diagnosed across 39 countries [13]. Incidences of progeria in the USA are estimated is 1 in 8 million births, based on the number of cases [15].

\section{Clinical features}

No clinical features present at birth, but within one to two year they begin to display the effect of accelerated aging means severe growth retardation is usually observed [16]. Within the first year, patients have short stature growth is distributed, and weight is more affected than height $[12,17]$. The common symptoms of HGPS is loss of eye brows and eye lashes which can observed in early childhood and due to receding hairline and Blading can also observed. Generally, these patient have facial character include micro-ganthia (small jaw), craniofacial disproportion, prominent eyes, scalp veins and alopecia (loss of hair), restricted joint mobility and severe premature atherosclerosis [7]. Within the 6 mo to $2 \mathrm{y}^{*}$, usually 
alopecia occurs and most probably between the ages of 2 to $3 \mathrm{y}$, blad with the exception of fine, downy hair develop in children $[12,17]$. Lipodystrophy can observe in early 6 mo of life, but may not become visible until 3-4 y of age. Lipodystrophy is the vanishing of subcutaneous fat and thinning of the skin and it is noted at 6 mo of the life which causes the blood vessel to be more visible and skin appears wrinkled and aged looking [12]. The limbs become thin and may development of stiff joint, coxavalga, and dystrophic nails. Other abnormalities include progressive resorption of bone, also called osteolysis. In this condition, there is a distinctive changes show by bones, for example, resorption of clavicles and terminal phallanges (acro-osteolysis). Aseptic necrosis of the head of the femur and hip dislocation is also common and irregular dentition, a thin and high-pitched voice, a pyriform (pear-shaped) thorax can observe $[18,19]$. Children with HGPS condition have normal mental and motor development and shown appropriate behaviour, and are very active and cheerful [12]. Lateral in life endocrine dysfunction and low conduction occurred [17]. 50\% affected patients suffer from insulin resistance without progression to diabetes mellitus, ability to develop sexual characteristics fail in HGPS affected children [20]. Though the initiation of specific abnormality differs considerably, the progression of atherosclerosis and concerns of health in children having HGPS [7, 12, 17, 21, 22]. Side by side diastolic and systolic blood pressure increases the stiffness of the blood vessels. Congestive heart failure and chest pain are common [20]. Myocardial infarction and stroke are the results of cardiac or cerebrovascular-related events [17]. All these events occur within the onset of the age seven [23]. Latest research studies have suggested that transient ischemic attacks and mini stroke occurs young in children [20]. Children with progeria usually die of heart attacks or strokes.

\section{Causes}

Lamin is intermediate filament protein which makes a network on the inner nuclear membrane [24]. Lamin is the main constituent of the nuclear envelope. It is $20-50 \mathrm{~nm}$ thin protein meshwork that interacts with various proteins and chromatin [25]. Intermediate filamentous lamina, which plays an essential role in maintaining the structural support of the nucleus and chromatin organization, DNA replication and mRNA transcription $[8,24]$. LMNA encodes laminins A and C that is lamin A and lamin B [8]. Lamin B causes generally lethal effect because it is expressed throughout development, including gastrulation whereas lamin $A$ is expressed only in differentiated tissues [26]. Laminopathies is a result of the defect in the lamin A. according to the classification of the HGPS progeria is one of the most severe of the 16 laminopathies [27].

Mutation is the molecular cause of the various laminopathies which are observed over 340 from 1000 different patients. The molecular causes which affect genes which are mainly encoding for lamins and these are associated with lamin post-translational modification or proteins which interacts with lamin. HGPS is caused by the de novo point mutation in the lamin A gene. Due to the mutation in Lamin A, it activates a cryptic splice donor site and it is mainly reasonable for the excision of the C-terminal part of the 11exon [2].

LMNA is a causative gene of progeria presents in chromosome no 1 which encodes laminins $\mathrm{A}$ and $\mathrm{C}$ [8]. LMNA gene contain exons 1-12 and mainly both lamin A/C contain 12 exon and due to the an alternative splice site in intron 10 , which gives to two different mRNA that codes for pre lamin A and lamin C. Lamin A is different from lamin $\mathrm{C}$ at the $\mathrm{C}$-terminal and it lacks final part of exon 10, 11 and 12 [9].
A

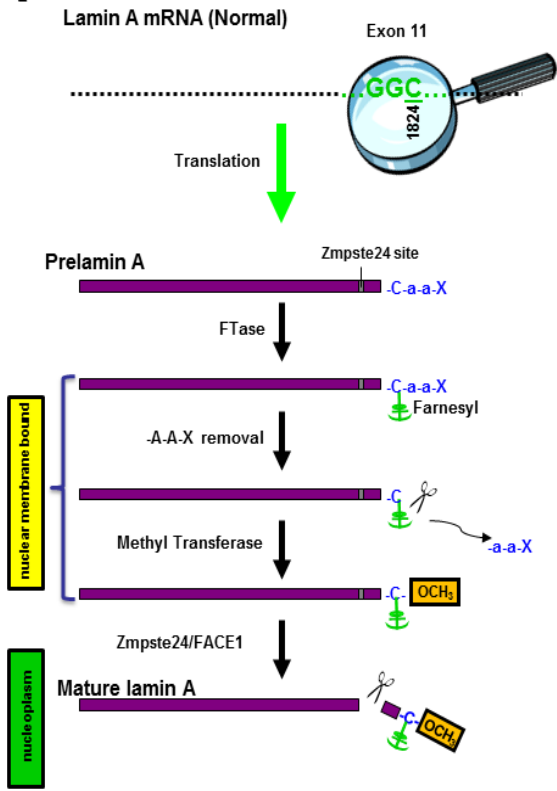

B

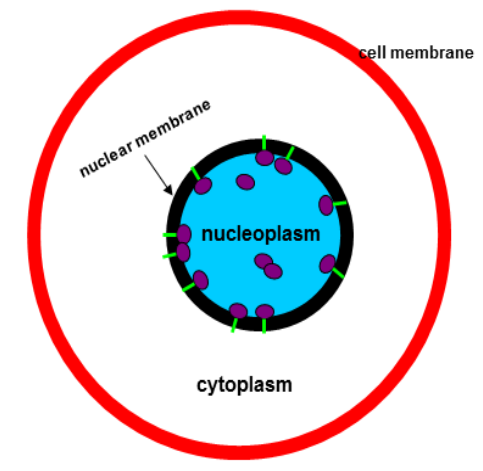

Fig. 1: This is the normal processing of lamin A from mRNA to mature lamin A. Normal translation of lamin A mRNA to prelamin A then prelamin A becomes farnesylated. The result of this farnesylated is anchoring to the inner nuclear membrane. Zmpste24/FACE1 cleavage of terminal amino acids results in release from the nuclear membrane and localization to the nucleoplasm. In normal processing, prelamin A is exists anchored to the nuclear membrane and then this is finally cleaved by Zmpste24/FACE1 then produce mature lamin A which mainly localizes to the nucleoplasm [28]

Due to the post-translational modification, Lamin $A$ is translated from LMNA as a precursor of prelamin $A$ the protein prelamin $A$ is responsible for the formation and maturation of the lamin $A$ [29]. The C-terminal ends in a group of 18 amino acid where the last four are CAAX cysteine, aliphatic, aliphatic any amino acid motif prompting prenylation such as tail is hallmark of farnesylated proteins. Prelamin $A$ undergoes a series of post-translational processing step. Prenylation generally occurs by the addition of a farnesyl group which is attached to the aysteine residue at the Cterminal in CAAX via farnesylation (farnesyltransferase). Second, the (-aax tri-peptide) the C-terminal 3 amino acid are enzymatically released by the endoprotease ZMPSTE24; third farnesyl cytenine is than methylated through methyltransferase [30]. In the last step of maturation, the $c$ terminal of 15 amino acids of prelamin $\mathrm{A}$ are 
removed again by a zinc metalloproteinase (Zmpste24; also known as FACE1), and finely mature lamin $\mathrm{A}$ is formed [31] The

A

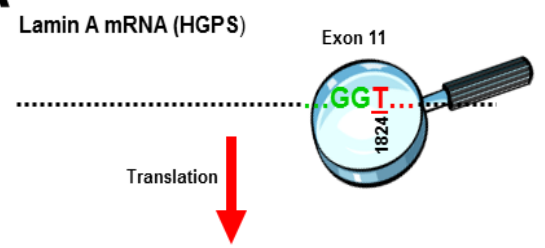

Prelamin A

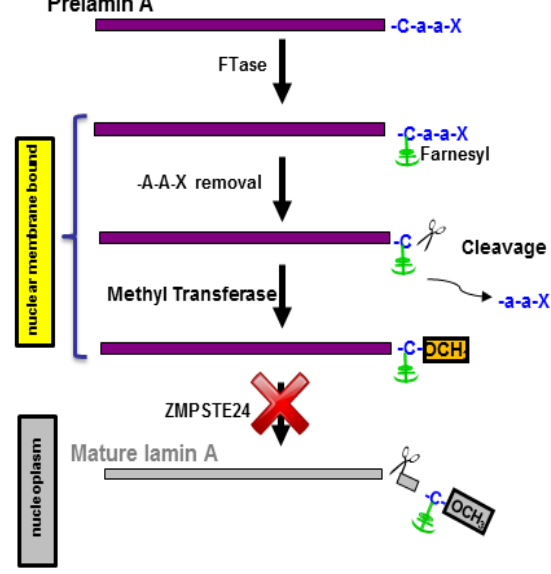

farnesylation increases lipophilicity and membrane association of lamin A [32].
B

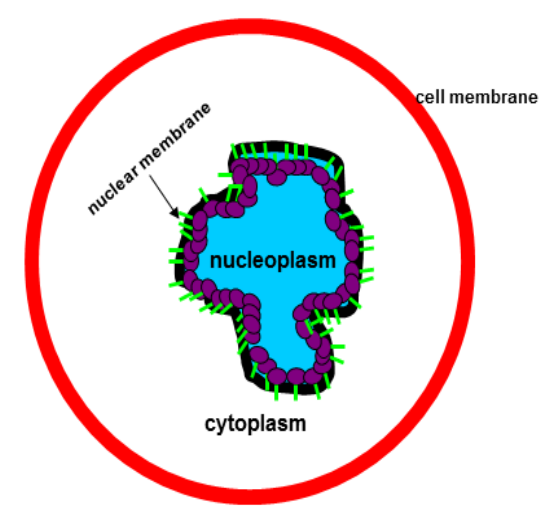

Fig. 2: But in case patient with progeria the processing of lamin $A$ in cells. Lamin is mutant and this mutated lamin $A$ mRNA is translated to prelamin A but that does not contain Zmpste24/FACE1 cleavage site. So, Prelamin A then becomes farnesylated resulting in anchoring to the inner nuclear membrane. Due to absence of Zmpste24 cleavage of terminal amino acids results in the inability to generate mature lamin A. In HGPS patients prelamin a remains farnesylated in the cell, that will accumulates at the inner nuclear membrane, and results in misshapen nuclei [28]

\section{CASE REPORT}

There is a number of cases observed in worldwide.

CASE 1: This is a case of the 14-year-old girl child, which is reported coarsening of skin, failure to thrive and inability to squat in her past history three to four years ago. His past history also showed developed global alopecia. But, perinatal history was uneventful. Till one year of life, she was normal when her parents started noticing the above-mentioned features. She had also normal intelligence. There were no family members were affected with these similar complaints. On general examination, the girl child showed physically short stature, malnourished, prominent eyes with hypoplastic chin, multiple patches on the skin, Coarse and thickening skin, mostly over the dorsum of the hands and shoulders are also observed. The fingers appeared broad and stubby at the terminal ends. Based on the history and clinical findings a provisional diagnosis of progeria was made. The Biochemical investigations of the patient were normal except serum cholesterol and urinary excretion of hyaluronic acid both are increased. For the confirmation of the disease, the child was subjected to a skeletal survey [33].

CASE 2: This is a case of progeria, which was reported in 1965, in this case, the patient were complaint hearing problem to pure-tone and speech audiometry, behavioural and electrophysiological auditory findings a young girl with progeria. A $5 \mathrm{y}^{*}$-old girl child with HGPS who presented typical characteristics of the disease, including short stature, alopecia, a small beak-like nose, micrognathia, and protruding ears. The diagnosis was made by a deys morphologist in the department of paediatrics. Computerized tomography showed a poorly pneumatized mastoid system. Otolaryngologic examination of the tympanic membrane and middle ear was unremarkable. A thorough otologic history, including information on possible middle ear disease, was not available. On the basis otolaryngology examination that is pure-tone, speech, immittance, and auditory brainstem response (ABR) audiometry is observed which suggest a 5-year-old female suffering from progeria. The female patient had a mild-to-moderate, conductive hearing loss. An immittance measurement is also done on the patient and that were consistent with fixation of the ossicular chain and this was mainly confirmed by surgically. Auditory central nervous system is also involved and that is mainly observed with mild prolonged ABR wave I-V latencies. [34].

CASE 3: This is a case study of a 12-year-old male patient that is reported to the clinic with a complaint of decayed both upper and lower anterior teeth region. Before two years ago patient's history with Clinical and radiographic features highly suggestive of HGPS and that is presented here with Description of differential diagnosis and dental consideration. His Past medical history confessed that the first two years of life were normal but as life followed day failure to gain both height and weight subsequently as well as followed by hair loss from scalp and eyebrows. Then stretching of skin and inability to stand or walk is also observed physically but, the patient was mentally normal. The child was already undergoing treatment for acute hepatitis. Before $2 y^{*}$ ago patient came to the hospital for the treatment of dental caries which is already explained his past dental history. But at that time Patient was uncooperative so, treatment could not be done [35].

CASE 4: Two siblings of Progeria patient are described in this case a 14-y-old male child and a 13-y-old female child. They have born to third-degree consanguineous parents. Both are showing patent anterior fontanalle, frontal bossing, and protruding ears. They had prominent eyes; beaked nose and micrognathia, high pitched voice. Their teeth were crowded and irregularly erupted. A 13-y-old female child showed sparse whereas in Male child eyebrows were absent. Their intelligence was normal. On the examination, the girl was found to an extremely short stature Height was $90 \mathrm{~cm}$ and weight $10 \mathrm{Kg}$. whereas in Male patient height is $91 \mathrm{~cm}$ and weight is 
$11 \mathrm{Kg}$. During both the pregnancies the antenatal period was uneventful were reported in Mother History. They both the siblings were delivered by cesarean section. On examination, their birth weight was more than $2.75 \mathrm{~kg}$. On dermatological examination revealed shiny hyperpigmented and wrinkled skin observed over forehead, hands and feet. Hypopigmented macules interspersed between hyperpigmented macules in the chest and abdomen are observed in both the siblings. Dystrophies of the finger and nails also observed. They had no photosensitivity. On biochemical examination, there was no evidence of primary endocrinological dysfunction.

Other biochemical analysis such as blood sugar, renal and liver parameters and lipid profile, blood count, hemoglobin percentage, Total thyroxine (TT4), thyroid stimulating hormone (TSH), growth hormone $(\mathrm{GH})$ were within normal ranges. Urine mucopolysaccharides were shows negative. USG abdomen shows a mild hepatomegaly in the male child whereas in female child no significant findings. ECG parameter was within normal ranges whereas Echo showed mild anterior mitral leaflet thickening and trivial aortic regurgitation in the male child patient whereas in female mitral valve prolapse with trivial mitral regurgitation but their left ventricular function was normal. On ophthalmologic examination, visual disturbances were found in the male patient. He was found to have Hypermetropia and pseudo papillitis. The female child had a complaint a swelling in the gums and it was diagnosed as peripheral ossifying fibroma in the dental OPD. On Radiographic examination, CT and X-ray skull revealed normal facial and skull bones with open anterior fontanalle. On histology examination, Skin biopsy showed thinned out epidermis and increased collagen in the mid-dermis. Diagnosis of progeria is basically based on the clinical features and biochemical examination of the patients which shows the short stature with bird-like facies, hair and nail changes and normal intelligence [36].

\section{Diagnosis}

Although the pursuit for finding an effective treatment for HGPS is still on, yet there is still no diagnostic clinical approved test to diagnose progeria [32]. Most of the cases of HGPS appear due to de novo point mutation in the same codon. Diagnosis of the progeria established by the following methods:

\section{Diagnostic methods}

To diagnose progeria, doctors observed phenotypes like symptoms that is physical symptoms, such as skin changes and a failure to gain weight, and as well as x-rays of patients and on the basis of the excretion of the glycosaminoglycan and urinary hyaluronic acid testing and radiography.

\section{$\checkmark \quad$ Imaging studies}

Diagnosis currently depends upon recognition of clinical and radiographic findings clinical diagnosis can also be established findings-diastasis of the sagittal suture with several wormier bones in the skull; hypoplastic mandible with infantile angle; the presence of fish mouth vertebrae; the occurrence of bilateral coxa valga deformity; resorption of terminal phalanges, etc [33]. The progressive bone loss from the distal phalanxes of the fingers and toes is one of the hallmarks of the disease [12-17].

$\checkmark$ Molecular diagnostic test: mutation screening is certainly theoretically feasible, especially with decreasing cost of genomic DNA analysis. Due to sporadic nature of the phenotype, predictive screening is not practically at present, since there is no way to determine which children at risk.

\section{$\checkmark$ Laboratory studies}

- There is abnormalities in serum lipid levels are limited to low high-density lipoprotein levels, which responsible for the atherosclerotic disease. Serum low-density lipoprotein and total cholesterol levels are normal in patients with (HGPS).

\section{Urinary hyaluronic test}

Elevated levels of hyaluronic acid excretion are seen in the urine of patients with HGPS but are not diagnostic. The significance is unknown [37].
After performing the urinary hyaluronic test on HGPS patient, hyaluronic acid level elevated in urine and decreased level of primary antioxidant enzymes in the blood as well as certain fatty acid compound. Due to decreased level of antioxidant enzymes in the blood, it may cause aging which believed to be a buildup oxidant in the blood. Urinary hylaronic is increased in most of the patient oh HGPS the measurement is now regarded as unreliable and is not recommended for the diagnosis [38]. Discovery of mutant lamin A gene, nowadays helpful for detection of the elevated mutant gene, that mutant gene identify from a blood sample and skin biopsy of the patient, this gives definitively diagnosis report. On other hand, the phenotypical evidence and medical history of a medical child this genetic test for lamin mutation is performed to confirm diagnose of HGPS to start the treatment programs early in the progression of the disorder [39].

\section{$\checkmark$ Prenatal testing}

Analysis of DNA extracted obtained by amino centesis from the fetal cell of HGPS children applied for prenatal diagnosis, mostly performed on about 15-18 $\mathrm{w}$ gestation or chronic villus sampling at about 10-12 w gestation. Before performing prenatal testing on effected family members must be identified for diseases causing alleles. For families pre-implantation genetic disorder may available through that disease-causing agent has been identified in effected family member [40].

\section{$\checkmark \quad$ Other tests}

For the detection of coronary artery disease and congestive heart failure, ECG and echocardiography are performed.

\section{Treatment}

To date, no specific therapy or effective treatment is available for HGPS. But cardiovascular and cerebral vascular component is essentially monitored. Aspirin is prescribed as prophylaxis to overcome the cardiovascular and cerebral vascular atherosclerotic diseases.

- To maintain physical activity and active lifestyle can be overcome with the help of physical and occupational therapy. By the use of hydrotherapy may be particularly effective in improving joint mobility and minimizing symptoms of arthritis.

- Infant with HGPS may have a poor feeding problem. So for the adequate nutrition gastronomy tube is inserted for supplemental enteral feeding.

For older children, they have taken too high energy supplements along with monitoring of growth and nutrition. Catabolic demands and sudden weight gain can be decreasing by growth hormone. Frneayl transferase inhibitor has a possible role in vitro of HGPS to promote initiate the release of pre-pro genin (mutanat prolamin A) from the nuclear membrane. It allows to be incorporated into nuclear lamina, which overcomes the structural and functional nuclear defect.

\section{CONCLUSION}

Progeria HGS (Hutchinson Gilford syndrome) is rare genetic disease characterized by segmental accelerated aging, rapid aging shortly after birth. It was originally described more than 100 y ago (HGPS) was named after Dr. Jonathan Hutchinson and Dr. Hasting Gliford; who described in England 1886 and 1897 respectively. HGPS is a rare disorder, involving a large number of different organ systems such as CNS, CVS, with a complex pathogenesis. The childhood disease is caused by a point mutation in the position of the LMNA gene by replacing cytosine with thymine, creating an unusual form of the protein lamin A. Nuclear envelope is made up of lamin A. This rare condition i. e remarkable because its symptoms strongly appear like normal human being, but occur in young children having symptoms; growth failure during the first y of life, narrow shrunken or wrinkled face, baldness, loss of eyebrows, lip dystrophy, scleroderma, decreased joint mobility, and a facial feature that resemble aged person. The most common HGPS mutation is located at codon $608(\mathrm{G} 608 \mathrm{G})$. This mutation creates a cryptic splice site within exon 11, which deletes a proteolytic cleavages site within the 
expressed mutant lamin A. Incomplete processing of pre lamin A results in nuclear abnormalities that can be observed in immune fluorescent studies of HGPS cells is presently no effective therapy is available for Hutchinson-Gilford progeria syndrome (HGPS). But, it is essential to monitor carefully cardiovascular and cerebrovascular disease. Low-dose aspirin is recommended for cardiovascular and cerebrovascular atherosclerotic disease.

\section{CONFLICTS OF INTERESTS}

\section{Declared none}

\section{REFERENCES}

1. Gilford H. On a condition of mixed premature and immature development. Med Chir Trans 1897;80:17-46.

2. Eriksson M, Brown WT, Gordon LB, Glynn MW, Singer J, Scott L, et al. Recurrent de novo point mutations in lamina cause Hutchinson-Gilford progeria syndrome. Nature 2003;423:293-8.

3. Gilford H. Ateleiosis and progeria: continuous youth and premature old age. Br Med J 1904;2:914-8.

4. Hutchinson J. Case of congenital absence of hair, the with atrophic condition of the skin and its appendages, in a boy whose mother had been almost wholly balk from alopeciaareata from the age of six. Lancet 1886;I:923.

5. Beauregard S, Gilchrest BA. Syndromes of premature aging. Dermatol Clin 1987;5:109-21.

6. Khalifa MM. Hutchinson-Gilford progeria syndrome: report of a Libyan family and evidence of autosomal recessive inheritance. Clin Genetics 1989;35:125-32.

7. DeBusk FL. The Hutchinson-Gilford progeria syndrome. J Pediatr 1972;90:697-724.

8. Shumaker DK, E Kuczmarski ER, Goldman RD. The nucleoskeleton: lamins and actin are major players in essential nuclear functions. Curr Opin Cell Biol 2003;15:358-66.

9. Lin F, Worman HJ. Structural organization of the human gene encoding nuclear lamin A and nuclear lamin C. J Biol Chem 1993;268:16321-6.

10. Sinensky M, Fantle K, Trujillo M, McLain T, Kupfer A, Dalton M. The processing pathway of prelamin A. J Cell Sci 1994;107:61-7.

11. Stuurman N, Heins S, Aebi U. Nuclear lamins: their structure, assembly, and interactions. J Struct Biol 1998;122:42-66.

12. Hennekam RCM. Hutchinson-Gilford progeria syndrome: a review of the phenotype. Am J Med Genet A 2006;140:2603-24.

13. Progeria Research Foundation (PRF) webpage. Available from: http://www.progeriaresearch.org/meet_the_kids.html. [Last accessed on 17 May 2014]

14. http://www.progeriaresearch.org/progeria_101. [Last accessed 26 Apr 2013]

15. Brown WT. Progeria: a human disease model of accelerated ageing. Am J Clin Nutr 1992;55:1222S-4S.

16. Sarkar PK, Shinton RA. Hutchinson-gilford progeria syndrome. Postgrad Med J 2001;907:312-7.

17. Merideth MA, Gordon LB, Clauss S, Sachdev V, Smith AC, Perry $\mathrm{MB}$, et al. Phenotype and course of Hutchinson-gilford progeria syndrome. N Engl J Med 2008;358:592-604.

18. Moen C. Orthopaedic aspects of progeria. J Bone Jt Surg 1982;64:542-6.

19. Gamble JG. Hip disease in Hutchinson-gilford progeria syndrome. J Pediatric Orthopaedics 1984;4:585-9.

20. Gordon LB, Brown WT, Collins FS, Pagon RA, Bird TD, Dolan CR, et al. Hutchinson-gilford progeria syndrome. Gene Rev 2011;45:421-38.

21. Baker PB, Baba N, Boesel CP. Cardiovascular abnormalities in progeria. Case report and review of the literature. Arch Pathol Lab Med 1981;105:384-6.
22. Al-Shali KZ, Hegele RA. Laminopathies and atherosclerosis. Arterioscler Thromb Vasc Biol 2004;24:1591-5.

23. Olive M, Harten I, Mitchell R, Beers JK, Djabali K, Cao K, et al. Cardiovascular pathology in Hutchinson-gilford progeria: correlation with the vascular pathology of aging. Arterioscler Thromb Vasc Biol 2010;30:2301-9.

24. Prokocimer M, Davidovich M, Nissim-Rafinia M, Wiesel-Motiuk N, Bar DZ, Barkan R, et al. Nuclear lamins: key regulators of nuclear structure and activities. J Cell Mol Med 2009;13:1059-85.

25. Burke B, Stewart CL. Life at the edge: the nuclear envelope and human disease. Nat Rev Mol Cell Biol 2002;3:575-85.

26. Vergnes L, Peterfy M, Bergo MO, Young SG, Reue K. Lamin B1 is required for mouse development and nuclear integrity. Proc Natl Acad Sci USA 2004;101:10428-33.

27. Zaremba-Czogalla $M$, Dubinska-Magiera $M$, Rzepecki $R$. Laminopathies: the molecular background of the disease and the prospects for its treatment. Cell Mol Biol Lett 2011;16:114-48.

28. Justin Parreno, BSc, MSca b, Alyssa V Cruz. BNc accelerated aging in patients with Hutchinson-gilford progeria syndrome: clinical signs, molecular causes, treatments, and insights into the aging process. UBCMJ 2011;3:9-10.

29. Davies BS, Fong LG, Yang SH, Coffinier C, Young SG. The posttranslational processing of prelamin a and disease. Annu Rev Genomics Hum Genet 2009;10:153-74.

30. Silvius JR, l'Heureux F. Fluorimetric evaluation of the affinities of isoprenylated peptides for lipid bilayers. Biochemistry 1994;15:3014-22.

31. Boban M, Braun J, Foisner R. Lamins: structure goes cycling. Biochem Soc Trans 2010;38:301-6.

32. Seema N, Dharti S, Puja N, Komal S, Kavita A, Shruti C, et al. A brief review MGM's medical college, N-6, CIDCO, Aurangabad. J Pharm Life Sci 2013;2965-2969, 2965.

33. Rastogi R, Chander Mohan SM. Progeria syndrome: a casereport. Indian J Orthop 2008;42:97-9.

34. James W, Hall III, James C. Denneny III, audiologic and otolaryngologic findings in progeria: case report. J Am Acad Audiol 1993;4:116-21.

35. Rajat GP, Antarmayee P, Poornima VK, Priyadarshini C, Sanat $\mathrm{KB}$, Ruchi $\mathrm{B}$, et al. Case report: Hutchinson-gilford progeria syndrome: a rare genetic disorder hindawi publishing corporation. Case Reports Dentistry; 2013. http://dx.doi.org/ $10.1155 / 2013 / 631378$

36. Sowmiya R, Prabhavathy D, Jayakumar S. Progeria in siblings: a rare case report. Indian J Dermatol 2011;56:581-2.

37. Gordon LB, McCarten KM, Giobbie-Hurder A. Disease progression in Hutchinson-gilford progeria syndrome: impact on growth and development. Pediatrics 2007;120:824-33.

38. Brown WT, Zebrower M, Kieras FJ. Progeria: a genetic disease model of premature aging. In: Harrison DE. Ed. Genetic Effects on Aging II; 1990. p. 521-42.

39. Gordon LB, Harten IA, Calabro A, Sugumaran G, Csoka AB, Brown WT, et al. Hyaluronan is not elevated in urine or serum in Hutchinson-gilford progeria syndrome. Hum Genet 2003;113:178-87.

40. Gray MD, Shen JC, Kamath-Loeb AS, Blank A, Sopher BL, Martin $\mathrm{GM}$, et al. The Werner syndrome protein is DNA helicase. Nat Genet 1997;17:100-3.

\section{How to cite this article}

- $\quad$ Savita Devi, Kamaldeep Singh. Risk factors, prevalence and diagnosis of Hutchinson Hutchinson gliford syndrome with special reference to case reports. Int J Pharm Pharm Sci 2017;9(5):1-5. 